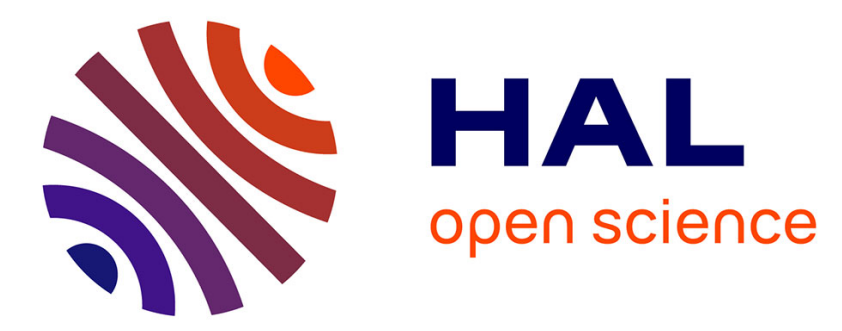

\title{
Stereoselective synthesis of enantiopure analogues of (-)-cephalotaxine
}

Farouk Berhal, Joelle Pérard-Viret, Jacques Royer

\section{To cite this version:}

Farouk Berhal, Joelle Pérard-Viret, Jacques Royer. Stereoselective synthesis of enantiopure analogues of (-)-cephalotaxine. Tetrahedron: Asymmetry, 2010, 21 (3), pp.325-332. 10.1016/j.tetasy.2010.01.004 . hal-03386006

\section{HAL Id: hal-03386006 https://hal.science/hal-03386006}

Submitted on 19 Oct 2021

HAL is a multi-disciplinary open access archive for the deposit and dissemination of scientific research documents, whether they are published or not. The documents may come from teaching and research institutions in France or abroad, or from public or private research centers.
L'archive ouverte pluridisciplinaire HAL, est destinée au dépôt et à la diffusion de documents scientifiques de niveau recherche, publiés ou non, émanant des établissements d'enseignement et de recherche français ou étrangers, des laboratoires publics ou privés. 


\title{
Stereoselective synthesis of enantiopure analogues of $(-)$-cephalotaxine
}

\author{
Farouk Berhal, Joëlle Pérard-Viret, Jacques Royer* \\ UMR 8638 CNRS-Université Paris Descartes, Faculté des Sciences Pharmaceutiques et Biologiques, 4 Avenue de l'Observatoire, 75270 Paris cedex 06, France
}

\begin{abstract}
A B S T R A C T
The asymmetric total synthesis of three analogues of (-)-cephalotaxine with structural modification of the aromatic ring was achieved in 16 steps and acceptable overall yields. The procedure used was quite similar to that reported by our group for the total synthesis of (-)-cephalotaxine in 2004. The enantiopure spiranic compound $\mathbf{4}$ is a common intermediate on which various aromatic groups can be introduced. Unexpected and interesting different chemical behaviors were observed during these syntheses.
\end{abstract}

\section{Introduction}

Cephalotaxine $\mathbf{1}$ is the most abundant alkaloid extracted from several types of Cephalotaxus, evergreen conifers and shrubs native from South-East Asia. This natural compound, first isolated in $1963,{ }^{1}$ does not exhibit any pronounced biological activity. Nevertheless, cephalotaxine is very attractive because it is the precursor of homoharringtonine $\mathbf{2}$ and harringtonine $\mathbf{3}$ two other alkaloids extracted from the same plants, which exhibit a strong antileukaemic activity, particularly against chronic myeloid leukemia (Fig. 1). ${ }^{2}$ Homoharringtonine is currently in phases II-III of clinical trials. $^{3}$

Numerous groups have been interested in the total synthesis of cephalotaxine, ${ }^{4}$ particularly our laboratory whose strategy consisted of the formation of the rings (C and $\mathbf{D})$ first by the preparation of an enantioselective 1-azaspiro[4,4]nonane compound<smiles></smiles>

$1:(-)-c e p h a l o t a x i n e$

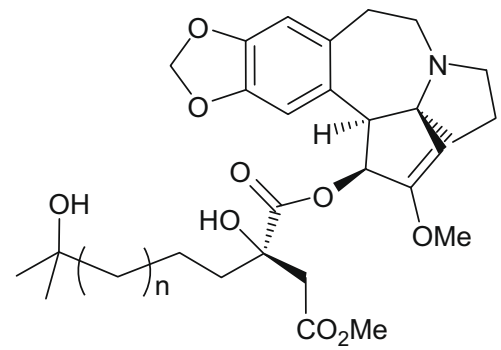

$2: \mathrm{n}=1$ homoharringtonine

$3: n=0$ harringtonine
Figure 1. Structures of (-)-cephalotaxine, homoharringtonine, and harringtonine.

\footnotetext{
* Corresponding author. Tel.: +33 153739749; fax: +33 143291403

E-mail address: jacques.royer@parisdescartes.fr (J. Royer).
}

(obtained from an $\alpha, \beta$-unsaturated- $\gamma$-lactam). The end of the synthesis was based on the Kuehne synthesis of cephalotaxine ${ }^{5}$ (Scheme 1).

The availability of natural or synthetic cephalotaxine has led to several groups investigating the preparation of analogues of homoharringtonine by modification of the side chain moiety through hemisynthesis. Despite the numerous analogues which have been prepared, until now none of them have shown an improvement of the antileukemic activity. ${ }^{6}$

We have noticed that very few analogues of homoharringtonine and cephalotaxine with molecular modification of the cephalotaxane core existed. The only analogues of cephalotaxine with this type of modification were recently prepared by Tietze et al. $^{7}$ and represented simplified structures of cephalotaxine and deoxyharringtonine in a racemic form, while we reported some original alkylated cephalotaxine derivatives. ${ }^{5}$

In this context and with a future objective to prepare analogues of homoharringtonine for biological evaluation, we herein report the asymmetric synthesis of the first non-natural analogues of cephalotaxine with modification at the aromatic ring.

The preparation of these analogues was accomplished by applying the total synthesis of cephalotaxine as described by our group in $2004 .^{5}$ The strategy consisted of the alkylation of the common spiranic compound $\mathbf{4}$ with different aromatic chains and then completing the sequence (Scheme 2).

\section{Results and discussion}

For the synthesis of the desired analogues it was necessary first to prepare the enantiopure spiranic compound 4 . Indeed this product represented the common intermediate in our strategy. It was easily synthesized in six steps from the commercially available (S)-1-(1-naphthyl)ethylamine ${ }^{8}$ and by using the chemistry of $\alpha, \beta-$ unsaturated- $\gamma$-lactams that has been developed for some years (Scheme 3). ${ }^{9}$ 


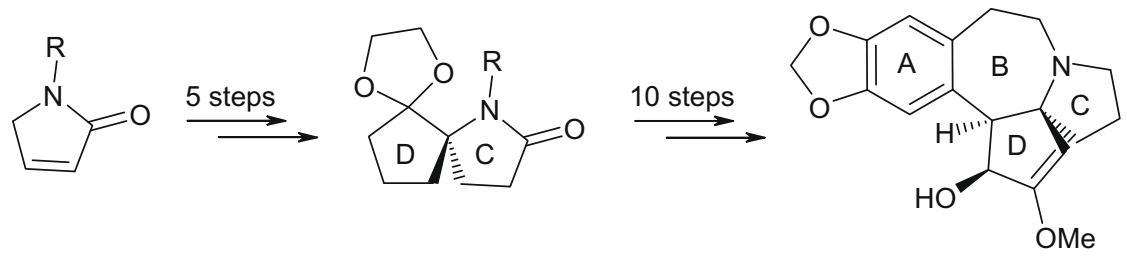

$1:(-)-$ cephalotaxine

Scheme 1. Strategy for the total synthesis of (-)-cephalotaxine.

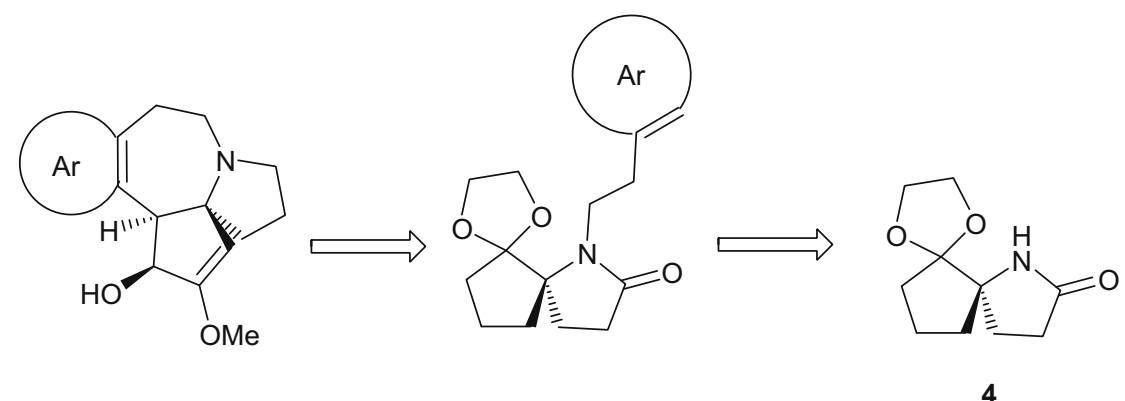

Ar : Aromatic ring

Scheme 2. Cephalotaxine analogues retrosynthetic sequence.

This spiranic compound 4 was thus obtained in 36\% overall yield over six steps and with an enantiomeric excess of about $99 \%$. The analogues were then synthesized following the sequence we already used for the total synthesis of cephalotaxine, with only minor changes.

Two analogues were first envisaged: one with a phenyl aromatic group and another one with a naphthyl aromatic group in place of the methylenedioxyphenyl group present in cephlotaxine. We observed some differences of reactivity according to the aromatic rings. The procedure used for the synthesis of cephalotaxine was reproduced in the phenyl and naphthyl series up to the cyclization leading to compound $\mathbf{9}$ (Scheme 4 ) and did not show important differences in their reactivities. The spiranic compound $\mathbf{4}$ reacted first with the appropriate aromatic ethyl tosylate and sodium hydride in refluxing benzene and gave the N-alkylated compounds 6a (74\% yield) and $\mathbf{6 b}$ (56\% yield). The keto group was deprotected to give $\mathbf{7}$, which was oxidized to enone $8 .^{10}$ The latter was selectively reduced ${ }^{11}$ to allylic alcohol prior to Friedel-Craft cyclization (Scheme 4). The two last steps were conducted without the isolation of the allylic alcohols and gave $\mathbf{9 a}(\mathrm{Ar}=\mathrm{Ph})$ and $\mathbf{9 b}$ $(\mathrm{Ar}=\mathrm{Naphth})$ in $71 \%$ and $54 \%$ yields, respectively. This showed that the cyclization does not require activation through electrodonating groups on the aromatic ring.
After the formation of the polycyclic core, the functionalization of the ring $\mathbf{D}$ was performed. A first difference of reactivity was observed during the dihydroxylation reaction of $\mathbf{9}$ to $\mathbf{1 0}$ (Scheme 5). In the synthesis of cephalotaxine, ${ }^{5}$ the reaction led to only one diastereomer. The dihydroxylation took place on the rear face of ring D of the cephalotaxane core (cf. Scheme 5) and in a good yield. This reaction showed that the configuration adopted by the polycyclic ring of cephalotaxine allowed the dihydroxylation to occur only on this face. When the same reaction was reproduced for analogue 9a $(\mathrm{Ar}=\mathrm{Ph})$, diol 10a was obtained in a good $88 \%$ yield but as a 9:1 mixture of two diastereomers. While this reaction was still highly diastereoselective it is noteworthy that the absence of the methylenedioxy group rendered the attack on the two faces of the ring $\mathbf{D}$ possible (even as a minor event).

The same conditions were then applied for the naphthyl aromatic group analogue $\mathbf{9 b}$. In this case, the same selectivity as in the cephalotaxine series was observed. In other words, only one diastereomer was formed and in high yield. These observations indicate that the substitution of the aromatic ring plays an important role on the geometry adopted by the cephalotaxane ring.

The separation of the two diastereomers $\mathbf{1 0 a}$ and $\mathbf{1 0 a} \mathbf{a}^{\prime}$ in the phenyl analogue series was possible but did not need to be carried out since the next step is the oxidation of the diols (Scheme 6). This

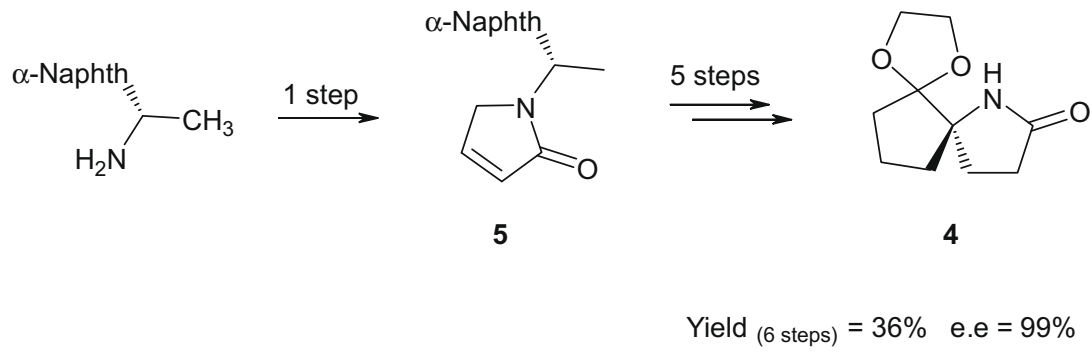

Scheme 3. Preparation of the spiranic compound. 

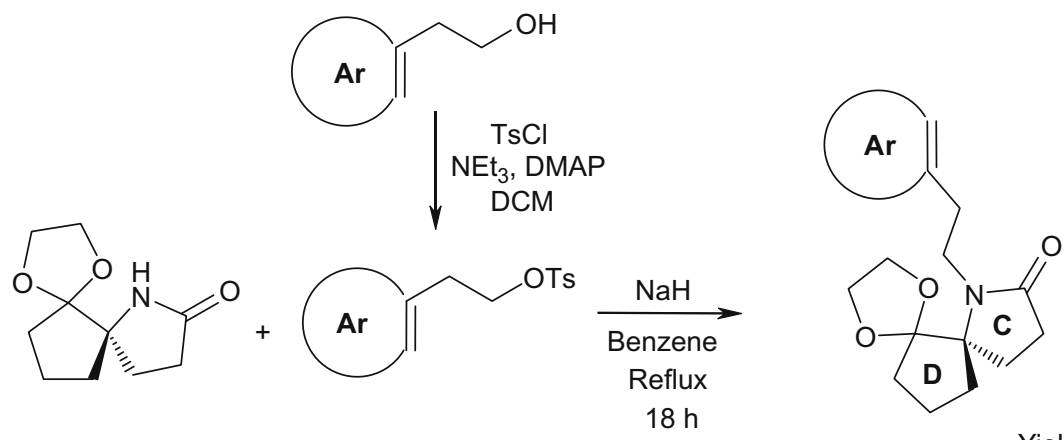

4
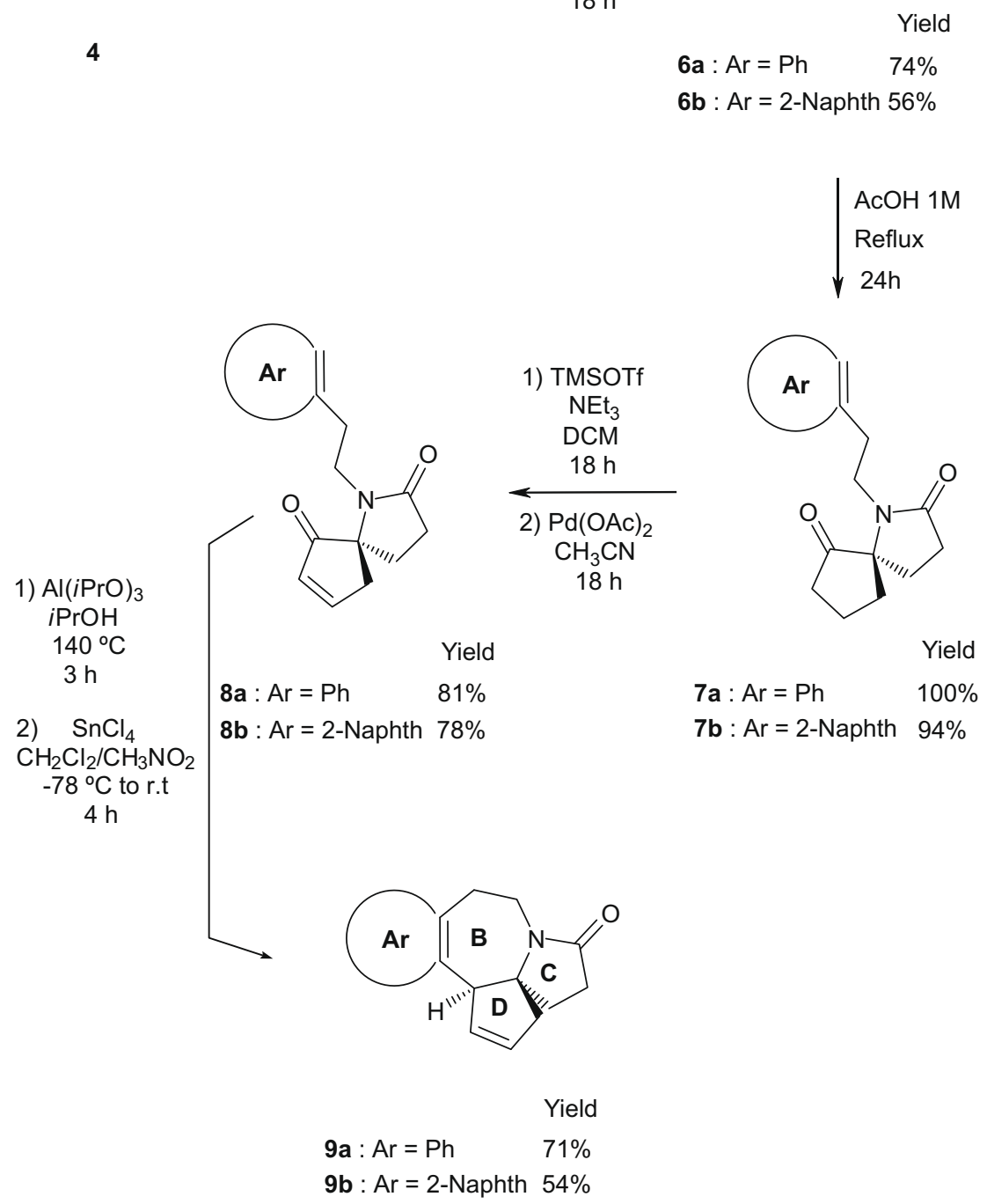

Scheme 4. General procedure for the analogues' synthesis.

reaction was straightforward and gave diones $\mathbf{1 1 a}$ and $\mathbf{1 1 b}$ in good yields only limited by the difficulty of the elimination of succinimide from the reaction mixture.

The further selective monomethylation to $\mathbf{1 2}$ showed several differences in the reactivity of diones $\mathbf{1 1}$ according to the aromatic ring (Scheme 6).

Kuehne's procedure ${ }^{4 \mathrm{f}}$ which was applied with success in the synthesis of cephalotaxine ( $\mathrm{MeOSiMe}_{3}, \mathrm{TfOH}$, and DCM) did not lead to the desired compound when applied to $\mathbf{1 1 a}$ or $\mathbf{1 1 b}$.

We then investigated other conditions. Several groups described this type of reaction in the total synthesis of cephalotaxine. We tried the conditions reported first by Weinreb ${ }^{4 \mathrm{~d}}$ and then modified by Fuchs. ${ }^{4 \mathrm{e}}$ Unfortunately by using the Weinreb-Fuchs method, the desired product was not obtained and the starting material was totally decomposed.

After several attempts it was possible to achieve this transformation using the Mori conditions ${ }^{4 t}$ with minor modifications (Scheme 7).

When the reaction was performed with the phenyl analogue 11a, the target molecule 12a was obtained with an optimized yield of $68 \%$. Over the course of the optimization of the reaction conditions, it was found that by increasing the amount of reactants, the regioisomer 13 was also formed in up to $38 \%$ yield.

The reaction was then applied to the naphthyl analogue $\mathbf{1 1 b}$. The conditions still needed to be optimized and the target molecule 12b was eventually obtained as a unique regioisomer in $60 \%$ yield. 


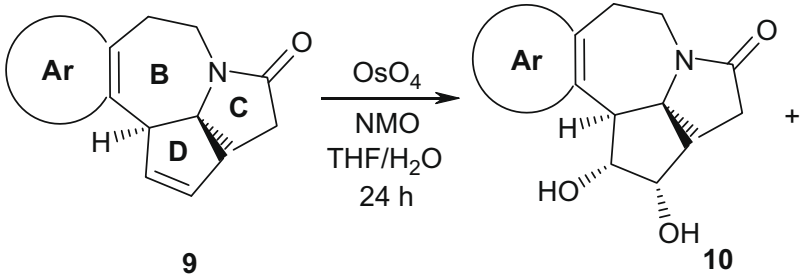

9

$$
\begin{aligned}
& \text { a } \mathrm{Ar}=\mathrm{Ph} \\
& \text { b } \mathrm{Ar}=\text { Naphth } \\
& \text { c } \mathrm{Ar}=3,4-\text { methylenedioxyphenyl }
\end{aligned}
$$

$$
\begin{array}{cccc}
90 & : & 10 & (88 \%) \\
100 & : & 0 & (93 \%) \\
100 & : & 0 & (81 \%)
\end{array}
$$

Scheme 5. Reactivity for the dihydroxylation reaction.

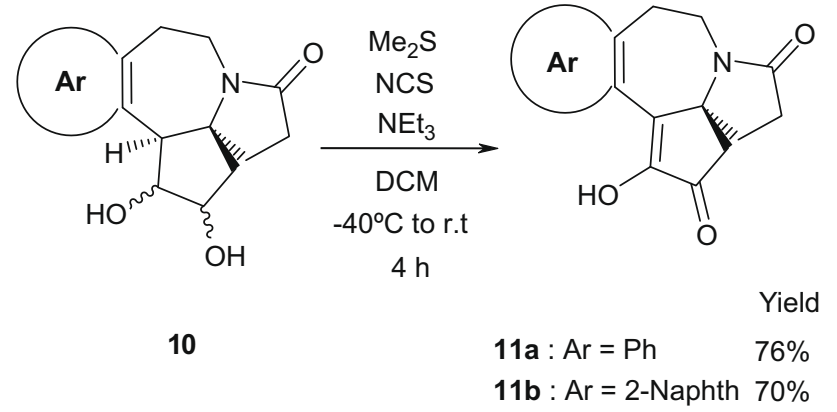

Scheme 6. Oxidation reactions.

Compounds 12a, 12b, and 13 could now be reduced to the target analogues via the reduction of the lactam and the ketone functions. This was achieved in a unique step using $\mathrm{AlH}_{3}$ in THF at low temperature (Scheme 8).

Finally, three analogues of cephalotaxine 14a, 14b, and 15 were prepared in 16 steps in good overall yields of $7 \%, 3 \%$, and $4 \%$, respectively (Fig. 2).

\section{Conclusion}

We were successful in the asymmetric synthesis of three analogues of cephalotaxine by using the same synthetic sequence as we had established for the natural product. The molecular diversity is concerned with the aromatic moiety and gives structures very close to the natural compound.

\section{Experimental}

\subsection{General}

Reagents were commercially obtained at highest commercial quality and used without further purification. All reactions were carried out under anhydrous conditions within an argon or nitrogen atmosphere in dry freshly distilled solvents. Reactions were monitored by thin-layer chromatography Merck 60F-254 precoated silica $(0.2 \mathrm{~mm})$ on aluminum. Flash chromatography was performed with SDS Silica Gel $60(35-70 \mu \mathrm{m})$. Melting points were determined on a Kofler apparatus and were not corrected. Specific rotations were measured at $25^{\circ} \mathrm{C}$ on a Perkin-Elmer 341 polarimeter with sodium ( $589 \mathrm{~nm}$ ) lamp. IR spectra were recorded using $\mathrm{NaCl}$ pellets on a Nicolet 205 spectrometer. Only noteworthy IR absorptions are listed. ${ }^{1} \mathrm{H}$ NMR and ${ }^{13} \mathrm{C}$ NMR spectra were recorded on a Bruker Avance-300 apparatus operating at 300 and $75 \mathrm{MHz}$, respectively. Chemical shifts were reported in $\delta$ (ppm).

4.2. Preparation of $(6 R)-7-p h e n e t h y l-1,4-d i o x a-7-a z a-d i s p i r o$ [4.0.4.3]tridecan-8-one (6a) and (6R)-7-(2-naphthalen-2-yl-ethyl)1,4-dioxa-7-aza-dispiro [4.0.4.3] tridecan-8-one (6b)

To a stirred solution of spiranic compound $\mathbf{4}$ ( 1 equiv) in an anhydrous benzene ( $c 0.29 \mathrm{M}$ ) was added in one portion sodium<smiles>CC(C)CC1CC2C(=O)C(O)=C3C4=C(CCCCCC4)CCN1C32</smiles>

11

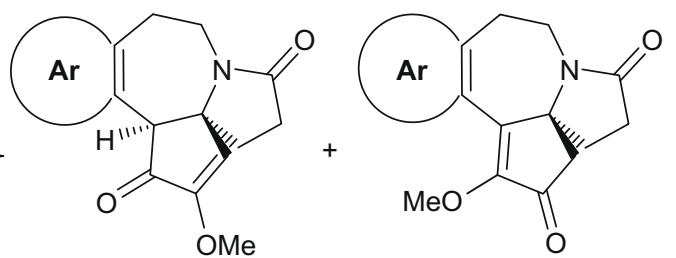

12
13

\begin{tabular}{lc|cc} 
a $\mathrm{Ar}=\mathrm{Ph}$ & $\mathrm{Ar}$ & conditions & product (Yield *) \\
\cline { 2 - 4 } b $\mathrm{Ar}=$ 2-Naphth & Phenyl & i & $\mathbf{1 2 a}(68 \%)$ \\
& Phenyl & ii & $12 \mathbf{a}(55 \%) / \mathbf{1 3}(38 \%)$ \\
& 2-Naphthyl & iii & $\mathbf{1 2 b}(60 \%)$
\end{tabular}

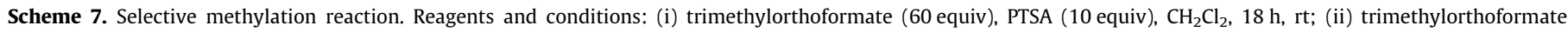
(80 equiv), PTSA (20 equiv), $\mathrm{CH}_{2} \mathrm{Cl}_{2}, 18 \mathrm{~h}$, rt; (iii) trimethylorthoformate (60 equiv), PTSA ( 10 equiv), $\mathrm{CH}_{2} \mathrm{Cl}_{2}$; reflux 48 h. * Isolated yields. 


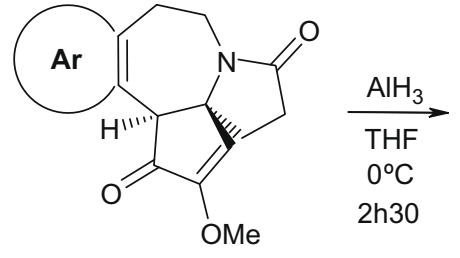

12

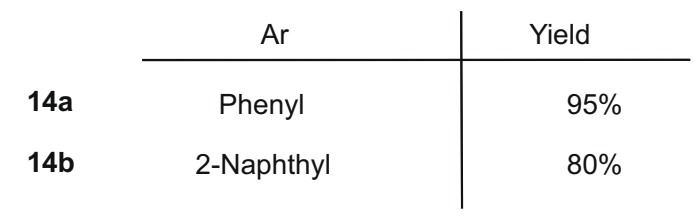<smiles>COC1=C2c3ccccc3CCN2C(=O)CCC1=O</smiles>

13

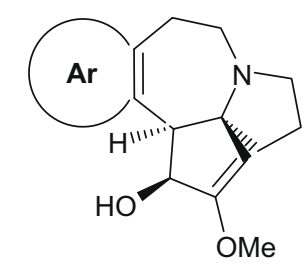

14

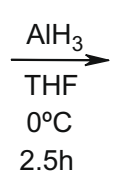<smiles>COC1=C2c3ccccc3CCN2CC[C@@H]1O</smiles>

$15(91 \%)$
Scheme 8. Reduction steps.

hydride (1.60 equiv). The suspension was stirred for $1 \mathrm{~h}$ at reflux then cooled to $\mathrm{rt}$ and the appropriate tosylate (1.80 equiv) was added in one portion. The suspension was stirred for $18 \mathrm{~h}$ at reflux, then cooled to rt and quenched with a saturated aqueous solution of ammonium chloride and the aqueous layer was extracted three times with dichloromethane. The organic layers were combined, dried on magnesium sulfate, evaporated under reduced pressure and the obtained residue was purified by flash chromatography on silica gel with AcOEt/cyclohexane $(80 / 20 \mathrm{v} / \mathrm{v})$ or $\mathrm{DCM} / \mathrm{MeOH}$ $(95 / 5 \mathrm{v} / \mathrm{v})$ as eluent to give the alkylated compound $\mathbf{6 a}(74 \%)$ as a white solid or $\mathbf{6 b}(56 \%)$ as a white solid.

Compound 6a: $\mathrm{mp} 84-86{ }^{\circ} \mathrm{C}$; $[\alpha]_{\mathrm{D}}^{23}=-52.6$ (c 1.125, $\left.\mathrm{CHCl}_{3}\right)$; IR (film) $v\left(\mathrm{~cm}^{-1}\right) 3019,2938,2875,1677 ;{ }^{1} \mathrm{H} \mathrm{NMR}\left(300 \mathrm{MHz}, \mathrm{CDCl}_{3}\right)$ : $\delta(\mathrm{ppm}) 1.17-1.31(\mathrm{~m}, 1 \mathrm{H}), 1.59-1.83(\mathrm{~m}, 4 \mathrm{H}), 1.87-2.01(\mathrm{~m}, 1 \mathrm{H})$, 2.03-2.16 (m, 1H), 2.23-2.39 (m, 2H), 2.62 (ddd, $J=9.3,11.0$, $16.8 \mathrm{~Hz}, 1 \mathrm{H}$ ), 2.79 (ddd, $J=7.0,10.0,12.8 \mathrm{~Hz}, 1 \mathrm{H}$ ), 2.93 (ddd, $J=4.8,9.8,13.0 \mathrm{~Hz}, 1 \mathrm{H}$ ), 3.43 (ddd, $J=4.7,9.8,12.9 \mathrm{~Hz}, 1 \mathrm{H}$ ), 3.74$3.90(\mathrm{~m}, 5 \mathrm{H}), 7.18-7.34(\mathrm{~m}, 5 \mathrm{H}) ;{ }^{13} \mathrm{C}$ NMR $\left(75 \mathrm{MHz}, \mathrm{CDCl}_{3}\right): \delta$ (ppm) 16.6, 30.3, 31.4, 32.6, 33.3, 35.0, 43.7, 64.6, 65.7, 71.2, $118.4,126.3,128.4,129.0,139.6,176.5$; MS (ESI) $\mathrm{m} / \mathrm{z} 324$ $\left([\mathrm{M}+\mathrm{Na}]^{+}\right), 340\left([\mathrm{M}+\mathrm{K}]^{+}\right)$; HRMS (ESI) calcd for $\mathrm{C}_{18} \mathrm{H}_{23} \mathrm{NO}_{3}$ $\left([\mathrm{M}+\mathrm{Na}]^{+}\right): 324.1570$; found: 324.1584 .

Compound 6b: $\mathrm{mp} 116-118{ }^{\circ} \mathrm{C} ;[\alpha]_{\mathrm{D}}^{23}=-29.9\left(\right.$ c $\left.0.835, \mathrm{CHCl}_{3}\right)$; IR (film) $v\left(\mathrm{~cm}^{-1}\right) 3050,2968,2942,2890,1675,1402 ;{ }^{1} \mathrm{H}$ NMR $\left(300 \mathrm{MHz}, \mathrm{CDCl}_{3}\right): \delta(\mathrm{ppm}) 1.22-1.30(\mathrm{~m}, 1 \mathrm{H}), 1.63-1.83(\mathrm{~m}, 4 \mathrm{H})$, $1.85-2.00(\mathrm{~m}, 1 \mathrm{H}), 2.08-2.21(\mathrm{~m}, 1 \mathrm{H}), 2.22-2.40(\mathrm{~m}, 2 \mathrm{H}), 2.58-$ $2.70(\mathrm{~m}, 1 \mathrm{H}), 2.94$ (ddd, $J=6.8,10.0,12.7 \mathrm{~Hz}, 1 \mathrm{H}$ ), 3.09 (ddd, $J=4.8,10.1,12.9 \mathrm{~Hz}, 1 \mathrm{H}$ ), 3.53 (ddd, $J=6.9,9.9,13.9 \mathrm{~Hz}, 1 \mathrm{H}$ ); 3.75-3.95 (m, 5H), 7.39-7.52 (m, 3H), $7.68(\mathrm{~s}, 1 \mathrm{H}), 7.76-7.90(\mathrm{~m}$, $3 \mathrm{H}) ;{ }^{13} \mathrm{C}$ NMR $\left(75 \mathrm{MHz}, \mathrm{CDCl}_{3}\right): \delta(\mathrm{ppm}) 16.6,30.3,31.4,32.6$, 33.5, 35.3, 43.6, 64.6, 65.7, 71.0, 118.3, 125.3, 126.0, 127.2, 127.5, 127.6, 127.7, 128.0, 132.1, 133.6, 137.0, 176.6; MS (ESI) $\mathrm{m} / \mathrm{z} 374$ $\left([\mathrm{M}+\mathrm{Na}]^{+}\right), 390\left([\mathrm{M}+\mathrm{K}]^{+}\right)$; HRMS (ESI) calcd for $\mathrm{C}_{22} \mathrm{H}_{25} \mathrm{NO}_{3}$ $\left([\mathrm{M}+\mathrm{Na}]^{+}\right)$: 374.1726; found: 374.171 .

\subsection{Preparation of (5R)-1-phenethyl-1-aza-spiro[4.4] nonane-2,6- dione 7a and (5R)-1-(2-naphthalen-2-yl-ethyl)-1-aza-spiro[4.4] nonane-2,6-dione $7 b$}

In a round-bottomed flask was placed compound $\mathbf{6 a}$ or $\mathbf{6 b}$ ( 1 equiv) in $1 \mathrm{M}$ acetic acid in water $(c 0.20 \mathrm{M})$. The homogeneous solution was stirred for $18 \mathrm{~h}$ at reflux, then cooled to $\mathrm{rt}$ and quenched with a saturated solution of sodium hydrogencarbonate and extracted three times with dichloromethane. The organic layers were combined, dried on magnesium sulfate, evaporated under reduced pressure and the obtained residue was purified by flash chromatography on silica gel with pure AcOEt as eluent to give the compound $\mathbf{7 a}(100 \%)$ as a clear oil or $\mathbf{7 b}(94 \%)$ as a white solid.

Compound 7a: $[\alpha]_{\mathrm{D}}^{23}=-16.5\left(\right.$ c $\left.0.945, \mathrm{CHCl}_{3}\right) ; \mathrm{IR}(\mathrm{NaCl}) v\left(\mathrm{~cm}^{-1}\right)$ 3058, 3027, 2961, 2938, 1750, 1688; $\left.{ }^{1} \mathrm{H} \mathrm{NMR} \mathrm{(300} \mathrm{MHz,} \mathrm{CDCl}_{3}\right): \delta$ (ppm) 1.72-2.23 (m, 7H), 2.35-2.56 (m, 3H), 2.83 (ddd, $J=5.3,10.1$, $13.3 \mathrm{~Hz}, 1 \mathrm{H}$ ), 2.89 (ddd, $J=6.4,10.0,13.3 \mathrm{~Hz}, 1 \mathrm{H}$ ), 3.11 (ddd, $J=6.6$, $10.1,13.8 \mathrm{~Hz}, 1 \mathrm{H}$ ), 3.47 (ddd, $J=5.3,10.0,13.8 \mathrm{~Hz}, 1 \mathrm{H}$ ), 7.19-7.33 $(\mathrm{m}, 5 \mathrm{H}) ;{ }^{13} \mathrm{C}$ NMR $\left(75 \mathrm{MHz}, \mathrm{CDCl}_{3}\right): \delta(\mathrm{ppm}) 17.2,28.9,29.6,33.2$, 34.9, 34.9, 43.3, 72.1, 126.4, 128.5, 128.9, 139.0, 175.8, 216.5; MS (ESI) $m / z 280\left([\mathrm{M}+\mathrm{Na}]^{+}\right), 296\left([\mathrm{M}+\mathrm{K}]^{+}\right)$; HRMS (ESI) calcd for $\mathrm{C}_{16} \mathrm{H}_{19} \mathrm{NO}_{2}$ ([M+Na] $\left.]^{+}\right)$: 280.1313; found: 280.1316 .

Compound 7b: $\mathrm{mp} 130^{\circ} \mathrm{C} ;[\alpha]_{\mathrm{D}}^{23}=-18.5$ (c $\left.0.920, \mathrm{CHCl}_{3}\right)$; IR (film) $v\left(\mathrm{~cm}^{-1}\right) 3054,2961,1747,1692 ;{ }^{1} \mathrm{H} \mathrm{NMR}\left(300 \mathrm{MHz}, \mathrm{CDCl}_{3}\right)$ : $\delta(\mathrm{ppm}) 1.68-1.94(\mathrm{~m}, 3 \mathrm{H}), 1.98-2.26(\mathrm{~m}, 4 \mathrm{H}), 2.36-2.59(\mathrm{~m}, 3 \mathrm{H})$, $2.90-3.14(\mathrm{~m}, 2 \mathrm{H}), 3.23$ (ddd, $J=6.3,10.2,13.2 \mathrm{~Hz}, 2 \mathrm{H}$ ), 3.54 (ddd, $J=5.2,10.2,15.8 \mathrm{~Hz}, 2 \mathrm{H}$ ), 7.35 (dd, $J=8.4,1.5 \mathrm{~Hz}, 1 \mathrm{H}$ ), 7.41-7.52 (m, 2H), $7.65(\mathrm{~s}, 1 \mathrm{H}), 7.74-7.87(\mathrm{~m}, 3 \mathrm{H}) ;{ }^{13} \mathrm{C}$ NMR $\left(75 \mathrm{MHz}, \mathrm{CDCl}_{3}\right): \delta(\mathrm{ppm}) 17.2,28.9,29.6,33.3,34.9,35.0,43.1$, 72.1, 125.4, 126.0, 127.1, 127.4, 127.4, 127.6, 128.1, 132.2, 133.6, 136.5, 175.8, 216.4; MS (ESI) $\mathrm{m} / z 330\left([\mathrm{M}+\mathrm{Na}]^{+}\right), 346\left([\mathrm{M}+\mathrm{K}]^{+}\right)$; HRMS (ESI) calcd for $\mathrm{C}_{20} \mathrm{H}_{21} \mathrm{NO}_{2}\left([\mathrm{M}+\mathrm{Na}]^{+}\right)$: 330.1465; found: 330.1473 .<smiles>COC1=C[C@@]23CCCN2CCc2ccc4ccccc4c2[C@H]1[C@@H]3O</smiles>

14b<smiles>COC1=C2c3ccccc3CCN2CC[C@@H]1O</smiles>

15

$4 \%$<smiles>COC1=C[C@@]23CCCN2CCc2ccccc2[C@H]1[C@H]3O</smiles>

$14 a$ 
4.4. Preparation of (5S)-1-phenethyl-1-aza-spiro[4.4] non-7ene-2,6-dione 8a and (5S)-1-(2-naphthalen-2-yl-ethyl)-1-azaspiro[4.4]non-7-ene-2,6-dione $8 b$

To a stirred solution of compound $\mathbf{7 a}$ or $\mathbf{7 b}$ ( 1 equiv) in an anhydrous dichloromethane $(c 0.10 \mathrm{M})$ at $0{ }^{\circ} \mathrm{C}$ was added triethylamine (1.60 equiv) and trimethylsilyltrifluoromethane sulfonate (1.50 equiv). The clear solution was stirred for $2 \mathrm{~h}$ at $0{ }^{\circ} \mathrm{C}$ and then for $16 \mathrm{~h}$ at room temperature. The solution was then quenched rapidly with cold distilled water and the aqueous phase was extracted three times with dichloromethane. The organic layers were combined, dried on magnesium sulfate, and evaporated under reduced pressure. The crude residue was then placed in a round-bottomed flask under argon, anhydrous acetonitrile $(c 0.10 \mathrm{M})$ and molecular sieves $4 \AA$ were added. The suspension was stirred for few minutes at room temperature and palladium diacetate (1.10 equiv for compound $\mathbf{7 a}$ and 1.63 equiv for compound $\mathbf{7 b}$ ) was added. The brown suspension was stirred at room temperature for $18 \mathrm{~h}$, then filtered on Celite ${ }^{\circledR}$ and the solids were washed with dichloromethane. The filtrate was evaporated under reduced pressure and the black obtained residue was purified by flash chromatography on silica gel with AcOEt/Cyclohexane $(80 / 20 \mathrm{v} / \mathrm{v})$ as eluent to give compound $\mathbf{8 a}$ ( $81 \%$ two steps) as a pale yellow oil or $\mathbf{8 b}$ ( $78 \%$ two steps) as a clear oil.

Compound 8a: $[\alpha]_{\mathrm{D}}^{23}=-293.6\left(\right.$ c 0.93, $\left.\mathrm{CHCl}_{3}\right)$; IR $(\mathrm{NaCl}) v\left(\mathrm{~cm}^{-1}\right)$ 3062, 3027, 2934, 1715, 1684; ${ }^{1} \mathrm{H}$ NMR (300 MHz, $\left.\mathrm{CDCl}_{3}\right): \delta(\mathrm{ppm})$ 1.90 (ddd, $J=6.6,9.3,12.7 \mathrm{~Hz}, 1 \mathrm{H}$ ), 2.25 (ddd, $J=6.2,9.6,12.7 \mathrm{~Hz}$, $1 \mathrm{H}), 2.51$ (ddd, $J=6.3,9.3,15.9 \mathrm{~Hz}, 1 \mathrm{H}), 2.59-2.81$ (m, 4H), 2.94$3.17(\mathrm{~m}, 1 \mathrm{H}), 3.16-3.26(\mathrm{~m}, 2 \mathrm{H}), 6.30(\mathrm{dt}, J=2.2,6.1 \mathrm{~Hz}, 1 \mathrm{H})$, $7.15-7.38(\mathrm{~m}, 5 \mathrm{H}), 7.71(\mathrm{dt}, J=2.7,5.7 \mathrm{~Hz}, 1 \mathrm{H}) ;{ }^{13} \mathrm{C} \mathrm{NMR}$ (75 MHz, $\left.\mathrm{CDCl}_{3}\right): \delta$ (ppm) 29.5, 31.2, 34.4, 40.6, 43.4, 68.6, 126.5, 128.5, 128.8, 126.5, 132.5, 139.1, 176.0, 207.9; MS (ESI) $m / z 278$ $\left([\mathrm{M}+\mathrm{Na}]^{+}\right), 294\left([\mathrm{M}+\mathrm{K}]^{+}\right)$; HRMS (ESI) calcd for $\mathrm{C}_{16} \mathrm{H}_{17} \mathrm{NO}_{2}$ $\left([\mathrm{M}+\mathrm{Na}]^{+}\right)$: 278.1157; found: 278.1166.

Compound 8b: $[\alpha]_{\mathrm{D}}^{23}=-138.6\left(c\right.$ 0.350, $\left.\mathrm{CHCl}_{3}\right)$; IR $(\mathrm{NaCl}) v$ $\left(\mathrm{cm}^{-1}\right)$ 3050, 3019, 2930, 1712, 1684, 1408, 1346; ${ }^{1} \mathrm{H}$ NMR $\left(300 \mathrm{MHz}, \mathrm{CDCl}_{3}\right): \delta$ (ppm) 1.82 (ddd, $\left.J=6.7,9.2,12.7 \mathrm{~Hz}, 1 \mathrm{H}\right)$, 2.15 (ddd, $J=6.2,9.5,12.7 \mathrm{~Hz}, 1 \mathrm{H}$ ), $2.36-2.50(\mathrm{~m}, 1 \mathrm{H}$ ), $2.52-2.68$ $(\mathrm{m}, 3 \mathrm{H}), 2.80-2.93(\mathrm{~m}, 1 \mathrm{H}), 3.04-3.35(\mathrm{~m}, 3 \mathrm{H}), 6.23(\mathrm{dt}, J=2.1$, 6.1, 1H), $7.27(\mathrm{dd}, J=1.7,8.4 \mathrm{~Hz}, 1 \mathrm{H}), 7.35-7.46(\mathrm{~m}, 2 \mathrm{H}), 7.58(\mathrm{~s}$, $1 \mathrm{H}), 7.61-7.65(\mathrm{~m}, 1 \mathrm{H}), 7.71-7.81(\mathrm{~m}, 3 \mathrm{H}) ;{ }^{13} \mathrm{C}$ NMR $(75 \mathrm{MHz}$, $\left.\mathrm{CDCl}_{3}\right): \delta$ (ppm) 29.6, 31.2, 34.5, 40.5, 43.2, 68.5, 125.5, 126.1, $127.1,127.3,127.4,127.6,128.1,132.1,133.5,136.5,132.4$, 162.7, 176.0, 207.8; MS (ESI) $\mathrm{m} / z 328\left([\mathrm{M}+\mathrm{Na}]^{+}\right), 344\left([\mathrm{M}+\mathrm{K}]^{+}\right)$; HRMS (ESI) calcd for $\mathrm{C}_{20} \mathrm{H}_{19} \mathrm{NO}_{2}\left([\mathrm{M}+\mathrm{Na}]^{+}\right)$: 328.1313 ; found: 328.131 .

\subsection{Preparation of $(4 S, 5 S)$-2,3-dehydro-15,16-desmethylen edioxy- cephalotaxan-8-one 9a and (4S,5S)-2,3-dehydro-19,20-desmethy- lenedioxy-14,19-benzo-cephalotaxan-8-one 9b}

To a stirred solution of compound $8 \mathbf{a}$ or $\mathbf{8 b}$ (1 equiv) in isopropanol ( $c 0.20 \mathrm{M}$ ) was added aluminum isopropo-xyde (30 equiv). The suspension was stirred at $140^{\circ} \mathrm{C}$ for $2 \mathrm{~h}$ with elimination of the solvent. The mixture was cooled to $\mathrm{rt}$ and an aqueous $1 \mathrm{M}$ $\mathrm{HCl}$ solution was added and the black suspension was stirred at room temperature for $15 \mathrm{~min}$. The aqueous phase was extracted three times with dichloromethane. The organic layers were combined, dried on magnesium sulfate, and evaporated under reduced pressure. The crude residue was then placed in a round-bottomed flask under argon, anhydrous dichloromethane and nitromethane ( $1: 1$ mixture, $c 0.050 \mathrm{M}$ ) were added. The solution was cooled at $-78^{\circ} \mathrm{C}$ and tin chloride ( 7.60 equiv) was slowly added. The pale yellow solution was stirred at $-78^{\circ} \mathrm{C}$ for $1.5 \mathrm{~h}$ and at rt for $2.5 \mathrm{~h}$, then an aqueous $1 \mathrm{M} \mathrm{HCl}$ solution was added and the aqueous layer was extracted two times with dichloromethane. The organic layers were combined, dried on magnesium sulfate, evaporated under reduced pressure and the obtained residue was purified by flash chromatography on silica gel with $\mathrm{CH}_{2} \mathrm{Cl}_{2} / \mathrm{MeOH}(95 / 5 \mathrm{v} / \mathrm{v})$ as eluent to give compound $\mathbf{9 a}$ (71\% two steps) as a white solid or $\mathbf{9 b}$ ( $54 \%$ two steps) as a white solid.

Compound 9a: $\operatorname{mp~} 100-106{ }^{\circ} \mathrm{C} ;[\alpha]_{\mathrm{D}}^{23}=-108.7\left(\right.$ c $\left.0.965, \mathrm{CHCl}_{3}\right)$; IR (film) $v\left(\mathrm{~cm}^{-1}\right) 3054,3043,2941,2868,1680 ;{ }^{1} \mathrm{H}$ NMR (300 MHz, $\left.\mathrm{CDCl}_{3}\right): \delta(\mathrm{ppm}) 2.06-2.30(\mathrm{~m}, 4 \mathrm{H}), 2.54-2.72(\mathrm{~m}, 2 \mathrm{H}$,), 2.92-3.11 (m, 2H), 3.39 (ddd, $J=8.2,11.2,14.4 \mathrm{~Hz}, 1 \mathrm{H}), 3.89-3.96$ $(\mathrm{m}, 1 \mathrm{H}), 4.17$ (ddd, $J=7.9,11.3,13.5 \mathrm{~Hz}, 1 \mathrm{H}), 5.65-5.70(\mathrm{~m}, 1 \mathrm{H})$, 5.87-5.93 (m, 1H), 7.08-7.23 (m, 4H); ${ }^{13} \mathrm{C} \mathrm{NMR} \mathrm{(75} \mathrm{MHz,} \mathrm{CDCl}_{3}$ ): $\delta$ (ppm) 30.0, 30.2, 35.3, 37.4, 44.1, 63.8, 69.7, 127.0, 128.3, $130.1,130.2,128.0,132.1,137.1,137.7,174.9$; MS (ESI) $m / z 240$ $\left([\mathrm{M}+\mathrm{H}]^{+}\right), 262\left([\mathrm{M}+\mathrm{Na}]^{+}\right), 278\left([\mathrm{M}+\mathrm{K}]^{+}\right)$; HRMS (ESI) calcd for $\mathrm{C}_{16} \mathrm{H}_{17} \mathrm{NO}\left([\mathrm{M}+\mathrm{Na}]^{+}\right)$: 262.1204; found: 262.1204 .

Compound 9b: mp $184-190^{\circ} \mathrm{C} ;[\alpha]_{\mathrm{D}}^{23}=+128.5$ ( c 0.755, $\left.\mathrm{CHCl}_{3}\right)$; IR (film) $v\left(\mathrm{~cm}^{-1}\right) 3043,2918,1684 ;{ }^{1} \mathrm{H}$ NMR $\left(300 \mathrm{MHz}, \mathrm{CDCl}_{3}\right): \delta$ (ppm) 1.84-2.00 (m, 1H), 2.11-2.40 (m, 3H), 2.67-2.84 (m, 2H), 3.04-3.25 (m, 2H), 3.67 (ddd, $J=8.0,11.8,15.2 \mathrm{~Hz}, 1 \mathrm{H}), 4.20$ (ddd, $J=7.5,11.7,13.4 \mathrm{~Hz}, 1 \mathrm{H}), 5.05-5.11(\mathrm{~m}, 1 \mathrm{H}), 5.67-5.75(\mathrm{~m}$, $1 \mathrm{H}), 5.96-6.05(\mathrm{~m}, 1 \mathrm{H}), 7.27(\mathrm{~d}, J=8.3 \mathrm{~Hz}, 1 \mathrm{H}), 7.43-7.61(\mathrm{~m}$, $2 \mathrm{H}), 7.73(\mathrm{~d}, J=8.3 \mathrm{~Hz}, 1 \mathrm{H}), 7.86(\mathrm{dd}, J=1.4,8.0 \mathrm{~Hz}, 1 \mathrm{H}), 8.22(\mathrm{~d}$, $J=8.6 \mathrm{~Hz}, 1 \mathrm{H}) ;{ }^{13} \mathrm{C}$ NMR $\left(75 \mathrm{MHz}, \mathrm{CDCl}_{3}\right): \delta(\mathrm{ppm}) 30.1,30.6$, $36.0,37.6,44.0,54.7,69.8,122.3,124.8,126.5,128.2,128.3$, 129.1, 129.2, 131.8, 132.4, 133.2, 135.9, 174.8; MS (ESI) $m / z 290$ $\left([\mathrm{M}+\mathrm{H}]^{+}\right), 312\left([\mathrm{M}+\mathrm{Na}]^{+}\right) ;$HRMS (ESI) calcd for $\mathrm{C}_{20} \mathrm{H}_{19} \mathrm{NO}$ $\left([\mathrm{M}+\mathrm{Na}]^{+}\right): 312.1364$; found: 312.1351 .

\subsection{Preparation of $(2 S, 3 R, 4 S, 5 S)-2,3$-dihydroxy-15,16-desmeth- ylenedioxy-cephalotaxan-8-one 10 a and $(2 R, 3 S, 4 S, 5 S)-2,3-$ dihydroxy-15,16-desmethylenedioxy-cephalotaxan-8-one 10a and $(2 S, 3 R, 4 S, 5 S)$-2,3-dihydroxy-19,20-desmethylenedioxy- 14,19-benzocephalotaxan-8-one 10b}

To a stirred solution of compound $9 \mathbf{a}$ or $\mathbf{9 b}$ ( 1 equiv) in a mixture of $\mathrm{THF} / \mathrm{H}_{2} \mathrm{O}(9 / 1)(c \quad 0.20 \mathrm{M})$ was added $\mathrm{N}$-methylmorpholine- $N$-oxide ( 1.50 equiv). The solution was stirred for few minutes and osmium tetroxyde ( 0.05 equiv) was added. The solution was stirred at room temperature for $24 \mathrm{~h}$, and then an aqueous $3 \mathrm{M} \mathrm{HCl}$ solution and a solution of sodium hydrogen sulfite (15\%) were added. The mixture was stirred at $\mathrm{rt}$ for $1 \mathrm{~h}$. The aqueous layer was extracted five times with dichloromethane and five times with ethyl acetate. The organic layers were combined, dried over magnesium sulfate, evaporated under reduced pressure, and the obtained residue was purified by flash chromatography on silica gel with $\mathrm{CH}_{2} \mathrm{Cl}_{2} / \mathrm{MeOH}(95 / 5 \mathrm{v} / \mathrm{v}$ ) as eluent to give compounds 10a and 10a' (88\% diastereomeric ratio: $90 / 10$ ) as a white solid or compound $\mathbf{1 0 b}(93 \%)$ as a white solid.

Compound 10a': ${ }^{1} \mathrm{H}$ NMR (300 MHz, $\left.\mathrm{CDCl}_{3}\right): \delta(\mathrm{ppm}) 1.81(\mathrm{dt}$, $J=12.8,9.7 \mathrm{~Hz}, 1 \mathrm{H}), 2.20-2.50(\mathrm{~m}, 3 \mathrm{H}), 2.20-2.50(\mathrm{~m}, 2 \mathrm{H}), 2.78$ (dd, $J=7.8,15.9,1 \mathrm{H}), 3.02-3.25(\mathrm{~m}, 2 \mathrm{H}), 3.38(\mathrm{dd}, J=3.8,11.5 \mathrm{~Hz}$, $1 \mathrm{H}), 3.76(\mathrm{~d}, J=4.0 \mathrm{~Hz}, 1 \mathrm{H}), 4.30(\mathrm{sl}, 1 \mathrm{H}), 4.68(\mathrm{dd}, 1 \mathrm{H}, J=8.5$, $14.8 \mathrm{~Hz}$ ), 7.02-7.38 (m, 4H).

Compound 10a: $\mathrm{mp} 220^{\circ} \mathrm{C}$; $[\alpha]_{\mathrm{D}}^{23}=+53.4\left(\mathrm{c} 0.955, \mathrm{CHCl}_{3}\right)$; IR (film) $v\left(\mathrm{~cm}^{-1}\right): 3478,3194,3062,2918,2883,1657 ;{ }^{1} \mathrm{H}$ NMR $\left(300 \mathrm{MHz}, \mathrm{CDCl}_{3}\right): \delta(\mathrm{ppm}) 2.04-2.46(\mathrm{~m}, 7 \mathrm{H}), 2.66(\mathrm{~s}, 1 \mathrm{H}), 2.74-$ $2.85(\mathrm{~m}, 1 \mathrm{H}), 3.05-3.24(\mathrm{~m}, 3 \mathrm{H}), 4.07-4.20(\mathrm{~m}, 1 \mathrm{H}), 4.32(\mathrm{t}$, $J=4.1 \mathrm{~Hz}, 1 \mathrm{H}), 4.45-4.53(\mathrm{~m}, 1 \mathrm{H}), 7.10-7.25(\mathrm{~m}, 4 \mathrm{H}) ;{ }^{13} \mathrm{C} \mathrm{NMR}$ $\left(75 \mathrm{MHz}, \mathrm{CDCl}_{3}\right): \delta(\mathrm{ppm}) 29.7,30.5,38.4,39.3,40.1,62.3,68.0$, 72.1, 78.2, 127.4, 128.2, 130.6, 131.4, 136.4, 137.2, 175.6; MS (ESI) $m / z 274\left([\mathrm{M}+\mathrm{H}]^{+}\right), 296\left([\mathrm{M}+\mathrm{Na}]^{+}\right), 310\left([\mathrm{M}+\mathrm{K}]^{+}\right)$; HRMS (ESI) calcd for $\mathrm{C}_{16} \mathrm{H}_{19} \mathrm{NO}_{3}\left([\mathrm{M}+\mathrm{Na}]^{+}\right)$: 296.1258; found: 296.1267 .

Compound 10b: $\mathrm{mp} 246-248{ }^{\circ} \mathrm{C} ;[\alpha]_{\mathrm{D}}^{20}=+177.9\left(\right.$ c $\left.1.06, \mathrm{CHCl}_{3}\right)$; IR (film) $v\left(\mathrm{~cm}^{-1}\right) 3420,3030,2930,2868,1649 ;{ }^{1} \mathrm{H}$ NMR (300 MHz, $\left.\mathrm{CDCl}_{3}\right): \delta(\mathrm{ppm}) 1.83-1.98(\mathrm{~m}, 1 \mathrm{H}), 2.04-2.18(\mathrm{~m}, 2 \mathrm{H})$, 2.24-2.50 (m, 3H), $2.63(\mathrm{~d}, J=5.2 \mathrm{~Hz}, 1 \mathrm{H}), 2.81(\mathrm{dd}, J=7.3$, 
$14.4 \mathrm{~Hz}, 1 \mathrm{H}), 2.97(\mathrm{~s}, 1 \mathrm{H}), 3.10-3.31(\mathrm{~m}, 2 \mathrm{H}), 3.90-4.15(\mathrm{~m}, 1 \mathrm{H})$; $4.27-4.34(\mathrm{~m}, 1 \mathrm{H}), 4.44(\mathrm{~d}, J=9.7 \mathrm{~Hz}, 1 \mathrm{H}), 4.55-4.65(\mathrm{~m}, 1 \mathrm{H})$, $7.26(\mathrm{~d}, J=8.3 \mathrm{~Hz}, 1 \mathrm{H}), 7.43-7.61(\mathrm{~m}, 2 \mathrm{H}), 7.73(\mathrm{~d}, J=8.3 \mathrm{~Hz}, 1 \mathrm{H})$, $7.84(\mathrm{~d}, J=8.0 \mathrm{~Hz}, 1 \mathrm{H}), 8.27(\mathrm{~d}, J=8.9 \mathrm{~Hz}, 1 \mathrm{H}) ;{ }^{13} \mathrm{C} \mathrm{NMR}(75 \mathrm{MHz}$, $\mathrm{CDCl}_{3}$ ): $\delta$ (ppm) 29.5, 30.8, 38.4, 39.3, 40.5, 52.8, 68.1, 72.1, 79.3, $122.8,125.2,126.8,128.3,128.8,128.9,133.1,133.2,133.4$, 134.5, 175.7; MS (ESI) $m / z 346\left([\mathrm{M}+\mathrm{Na}]^{+}\right), 362\left([\mathrm{M}+\mathrm{K}]^{+}\right)$; HRMS (ESI) calcd for $\mathrm{C}_{20} \mathrm{H}_{21} \mathrm{NO}_{3}\left([\mathrm{M}+\mathrm{Na}]^{+}\right)$: 346.1414 ; found: 346.1418 .

4.7. Preparation of (5S)-3,4-dehydro-3-hydroxy-15,16-desmethylenedioxy-cephalotaxan-2,8-dione 11 a and (5S)-3,4-dehydro3-hydroxy-19,20-desmethylenedioxy-14,19-benzocephalotaxan2,8-dione 11b

In a round-bottomed flask was placed under argon $N$-chlorosuccinimide ( 5 equiv) in anhydrous dichloromethane $(c 0.83 \mathrm{M})$. The solution was cooled at $0{ }^{\circ} \mathrm{C}$ and dimethylsulfide ( 5 equiv) was added. The white suspension obtained was stirred for $30 \mathrm{~min}$ at $0{ }^{\circ} \mathrm{C}$, then cooled at $-40^{\circ} \mathrm{C}$ and a solution of compounds 10a and $\mathbf{1 0 \mathbf { a } ^ { \prime }}$ or 10b (1 equiv) in an anhydrous dichloromethane (c $\left.0.04 \mathrm{M}\right)$ was added dropwise. The suspension was stirred at $-40^{\circ} \mathrm{C}$ for $1 \mathrm{~h}$ and triethylamine ( 8 equiv) was added in one portion. The clear solution was placed at $\mathrm{rt}$ and stirred for $1.5 \mathrm{~h}$. A solution of saturated sodium chloride was added and the aqueous phase was extracted two times with dichloromethane. The organic layers were combined, dried over magnesium sulfate, evaporated under reduced pressure and the obtained residue was purified by recrystallization in toluene (three times) and in water (two times) to give compound 11a (76\%) as a white solid or $\mathbf{1 1 b}(70 \%)$ as a white solid.

Compound 11a: $\mathrm{mp} 268^{\circ} \mathrm{C}$ (dec.); IR (film) $v\left(\mathrm{~cm}^{-1}\right) 3401,3070$, 2938, 2922, 1719, 1661, 1388; ${ }^{1} \mathrm{H}$ NMR (300 MHz, DMSO): $\delta$ (ppm) 1.71-1.84 (m, $1 \mathrm{H}), 1.86-1.97(\mathrm{~m}, 1 \mathrm{H}), 2.2(\mathrm{dd}, J=8.6,16.2 \mathrm{~Hz}, 1 \mathrm{H})$; $2.39-2.50(\mathrm{~m}, 1 \mathrm{H}), 2.72(\mathrm{AB}, J=17.6 \mathrm{~Hz} \Delta \delta=0.23 \mathrm{ppm}, 2 \mathrm{H}), 2.95-$ $3.25(\mathrm{~m}, 3 \mathrm{H}), 3.72-3.86(\mathrm{~m}, 1 \mathrm{H}),, 7.20-7.40(\mathrm{~m}, 4 \mathrm{H}) ;{ }^{13} \mathrm{C} \mathrm{NMR}$ (75 MHz, DMSO): $\delta$ (ppm) 30.0, 33.5, 33.7, 35.3, 44.8, 67.4, 126.2, 129.0, 130.5, 131.0, 136.7, 142.1, 150.4, 173.4, 199.5; MS (ESI) m/ $z 270\left([\mathrm{M}+\mathrm{H}]^{+}\right), 292\left([\mathrm{M}+\mathrm{Na}]^{+}\right)$; HRMS (ESI) calcd for $\mathrm{C}_{16} \mathrm{H}_{15} \mathrm{NO}_{3}$ $\left([\mathrm{M}+\mathrm{Na}]^{+}\right)$: 292.0950; found: 292.0944 .

Compound 11b: ${ }^{1} \mathrm{H}$ NMR (300 MHz, DMSO): $\delta$ (ppm) 1.90-2.08 (m, 1H), 2.10-2.15 (m, 1H), 2.20-2.50 (m, 2H), $2.72(\mathrm{AB}, J=18 \mathrm{~Hz}$ $\Delta \delta=0.18 \mathrm{ppm}, 2 \mathrm{H}), 3.15-3.45(\mathrm{~m}, 3 \mathrm{H}), 4.05-4.25(\mathrm{~m}, 1 \mathrm{H}), 7.20-$ $7.40(\mathrm{~m}, 1 \mathrm{H}), 7.42-7.57(\mathrm{~m}, 2 \mathrm{H}), 7.60-7.68(\mathrm{~m}, 1 \mathrm{H}), 7.75-7.95(\mathrm{~m}$, $2 \mathrm{H}) ;{ }^{13} \mathrm{C}$ NMR (75 MHz, DMSO): $\delta$ (ppm) 30.0, 32.8, 33.1, 35.1, 44.3, 66.7, 125.9, 126.0, 127.1, 128.5, 128.7, 129.0, 131.0, 132.1, 134.8, 139.9, 150.8, 173.7, 199.5; MS (ESI) $\mathrm{m} / z 342\left([\mathrm{M}+\mathrm{Na}]^{+}\right)$; HRMS (ESI) calcd for $\mathrm{C}_{20} \mathrm{H}_{17} \mathrm{NO}_{3}\left([\mathrm{M}+\mathrm{H}]^{+}\right)$: 320.1282 ; found: 320.1276 .

\subsection{Preparation of $(4 S, 5 S)$-1,2-dehydro-2-methoxy-15,16-des $\mathrm{m}$ ethylenedioxy-cephalotaxan-8-one 12a and (5S)-3,4-dehydro-3- methoxy-15,16-desmethylenedioxy-cephalotaxan-2,8-dione 13}

To a stirred solution of compound 11a (1 equiv) in anhydrous dichloromethane $(c 0.05 \mathrm{M})$ and under argon were added trimethylorthoformate (60 equiv) and para-toluene sulfonic acid (10 equiv). The pale yellow solution was stirred at room temperature for $18 \mathrm{~h}$, then distilled water was added and the phases were separated. The aqueous layer was extracted with dichloromethane, the organic layers were combined, washed three times with distilled water then dried over magnesium sulfate, evaporated under reduce pressure and the residue obtained was purified by flash chromatography on silica gel with AcOEt/MeOH (95/5 v/v) as eluent to give compound 12a (68\%) as a white solid. When the same reaction was made using trimethylorthoformate (80 equiv) and para-toluene sulfonic acid (20 equiv) at rt and for $18 \mathrm{~h}$ compound 12a was isolated with $55 \%$ yield and compound 13 with $38 \%$ yield as a white solid.
Compound 13: ${ }^{1} \mathrm{H}$ NMR (300 MHz, $\mathrm{CDCl}_{3}$ ): $\delta$ (ppm) 1.86-2.10 (m, $2 \mathrm{H}), 2.23$ (ddd, $J=1.4,8.8,16.5 \mathrm{~Hz}, 1 \mathrm{H}), 2.44-2.58(\mathrm{~m}, 1 \mathrm{H})$, $2.70(\mathrm{AB}, J=17.6 \mathrm{~Hz} \Delta \delta=1 \mathrm{ppm}, 2 \mathrm{H}), 3.08-3.24(\mathrm{~m}, 2 \mathrm{H}), 3.25-$ $3.37(\mathrm{~m}, 1 \mathrm{H}), 3.88(\mathrm{~s}, 3 \mathrm{H}), 4.06-4.18(\mathrm{~m}, 1 \mathrm{H}), 7.22-7.38(\mathrm{~m}, 4 \mathrm{H})$; ${ }^{13} \mathrm{C}$ NMR $\left(75 \mathrm{MHz}, \mathrm{CDCl}_{3}\right): \delta(\mathrm{ppm}) 30.0,33.2,35.4,45.6,58.9$, 66.9, 126.1, 129.4, 130.0, 130.8, 129.7, 136.3, 147.8, 151.6, 174.0, 198.3.

Compound 12a: $[\alpha]_{\mathrm{D}}^{23}=-78.7$ (c 0.915, $\mathrm{CHCl}_{3}$ ); IR (film) $v$ $\left(\mathrm{cm}^{-1}\right) 3085,3015,2965,2871,1723,1680,1637,1412 ;{ }^{1} \mathrm{H}$ NMR (300 MHz, $\left.\mathrm{CDCl}_{3}\right): \delta$ (ppm) 2.11-2.39 (m, 4H), 2.50-2.73 (m, 2H), 3.00 (dd, $J=7.0,13.3 \mathrm{~Hz}, 1 \mathrm{H}), 3.64(\mathrm{~s}, 1 \mathrm{H}), 3.86$ (s, 3H), 4.15 (ddd, $J=8.2,11.9,13.4 \mathrm{~Hz}, 1 \mathrm{H}), 6.11(\mathrm{~s}, 1 \mathrm{H}), 7.10-7.30(\mathrm{~m}, 4 \mathrm{H}) ;{ }^{13} \mathrm{C}$ NMR (75 MHz, $\left.\mathrm{CDCl}_{3}\right): \delta$ (ppm) 29.7, 31.1, 34.6, 38.0, 57.8, 63.2, $66.2,123.5,127.5,129.1,130.2,131.9,134.3,135.8,159.3,174.3$, 198.0; MS (ESI) $m / z 284\left([\mathrm{M}+\mathrm{H}]^{+}\right), 306\left([\mathrm{M}+\mathrm{Na}]^{+}\right)$; HRMS (ESI) calcd for $\mathrm{C}_{17} \mathrm{H}_{17} \mathrm{NO}_{3}\left([\mathrm{M}+\mathrm{Na}]^{+}\right)$: 306.1106; found: 306.1105 .

\subsection{Preparation of $(4 S, 5 S)$-1,2-dehydro-2-methoxy-19,20- desmethylenedioxy-14,19-benzocephalota xan-8-one 12b}

To a stirred solution of compound 11b (1 equiv) in anhydrous dichloromethane (c $0.07 \mathrm{M}$ ) and under argon were added trimethylorthoformate (60 equiv) and para-toluene sulfonic acid (10 equiv). The pale yellow solution was stirred at reflux for $48 \mathrm{~h}$. After cooled at room temperature, a solution of saturated sodium hydrogencarbonate was added and the phases were separated. The aqueous layer was extracted twice with dichloromethane. The organic layers were combined, dried over magnesium sulfate, evaporated under reduce pressure and the residue obtained was purified by flash chromatography on silica gel with AcOEt/MeOH $(95 / 5 \mathrm{v} / \mathrm{v})$ as eluent to give the compound 12b $(61 \%)$ as a white solid. Compound 12b: $[\alpha]_{\mathrm{D}}^{23}=+59.0$ (c 1.0, $\left.\mathrm{CHCl}_{3}\right) ;{ }^{1} \mathrm{H}$ NMR $\left(300 \mathrm{MHz}, \mathrm{CDCl}_{3}\right): \delta(\mathrm{ppm}) 1.88-1.97(\mathrm{~m}, 1 \mathrm{H})$, $2.20-2.40(\mathrm{~m}, 3 \mathrm{H}), 2.76-2.82(\mathrm{~m}, 2 \mathrm{H}), 3.07-3.11(\mathrm{~m}, 1 \mathrm{H}), 3.92$ (s, 3H), 4.13-4.25 (m, 1H), $4.75(\mathrm{~s}, 1 \mathrm{H}), 6.18(\mathrm{~s}, 1 \mathrm{H}), 7.3(\mathrm{~d}$, $J=8.3 \mathrm{~Hz}, 1 \mathrm{H}), 7.44-7.59(\mathrm{~m}, 2 \mathrm{H}), 7.80(\mathrm{~d}, J=8.2 \mathrm{~Hz}, 1 \mathrm{H}), 7.87$ $(\mathrm{dd}, J=1.4,8.0 \mathrm{~Hz}, 1 \mathrm{H}), 8.20(\mathrm{~d}, J=8.4 \mathrm{~Hz}, 1 \mathrm{H}) ; .{ }^{13} \mathrm{C}$ NMR $\left(75 \mathrm{MHz}, \mathrm{CDCl}_{3}\right): \delta$ (ppm) 29.5, 31.8, 34.5, 37.9, 55.8, 57.8, $66.4,121.5,125.3,127.2,128.3,129.3,129.5,123.1,129.8$, 133.1, 133.2, 134.5, 159.5, 174.4, 199.3; MS (ESI) $\mathrm{m} / \mathrm{z} 356$ $\left([\mathrm{M}+\mathrm{Na}]^{+}\right), \quad 372\left([\mathrm{M}+\mathrm{K}]^{+}\right)$; HRMS (ESI) calcd for $\mathrm{C}_{21} \mathrm{H}_{19} \mathrm{NO}_{3}$ $\left([\mathrm{M}+\mathrm{Na}]^{+}\right)$: 356.1263; found: 356.1266.

4.10. Preparation of $(3 S, 4 S, 5 S)$-1,2-dehydro-2-methoxy-3-hydroxy-15,16-desmethylenedioxy cephalotaxane 14 a and $(3 S, 4 S, 5 S)$ 1,2-dehydro-2-methoxy-3-hydroxy-19,20-desmethylenedioxy14,19-benzo-cephalotaxane 14b

To a stirred solution of $\mathrm{AlH}_{3} 1 \mathrm{M}$ in anhydrous THF (48 equiv) (previously prepared from $\mathrm{LiAlH}_{4}$ and $\mathrm{AlCl}_{3}$ ) was added dropwise at $0{ }^{\circ} \mathrm{C}$ a solution of compound 12a or $\mathbf{1 2 b}$ (1 equiv) in anhydrous THF ( $c 0.065 \mathrm{M}$ ) and stirred at $0{ }^{\circ} \mathrm{C}$ for $2.5 \mathrm{~h}$. The solution was then warmed to rt and a $2 \mathrm{M}$ solution of Rochelle's salt was added and stirred for $15 \mathrm{~min}$. The aqueous phase was extracted twice with dichloromethane. The organic layers were combined, dried over magnesium sulfate, evaporated under reduce pressure and the residue obtained was purified by flash chromatography on silica gel with AcOEt/MeOH/NH $/ \mathrm{NH}_{4} \mathrm{OH}(95 / 5 / 0.5 \mathrm{v} / \mathrm{v})$ as eluent to give the compound 14a (95\%) as a colorless oil or compound $14 \mathbf{b}(80 \%)$ as a colorless oil.

Compound 14a: $[\alpha]_{\mathrm{D}}^{23}=-150.4$ (c $\left.0.75, \mathrm{CHCl}_{3}\right) ;{ }^{1} \mathrm{H} \quad \mathrm{NMR}$ $\left(300 \mathrm{MHz}, \mathrm{CDCl}_{3}\right): \delta(\mathrm{ppm}) 1.63(\mathrm{~s}, 1 \mathrm{H}), 1.69-1.83(\mathrm{~m}, 2 \mathrm{H}), 1.85-$ $1.98(\mathrm{~m}, 1 \mathrm{H}), 1.99-2.12(\mathrm{~m}, 1 \mathrm{H}), 2.49(\mathrm{dd}, J=7.1,14.2 \mathrm{~Hz}, 1 \mathrm{H})$, 2.55-2.78 (m, 2H), 2.89-3.10 (m, 2H), 3.46 (ddd, $J=8.2,12.1$, $13.8 \mathrm{~Hz}, 1 \mathrm{H}), 3.74-3.80(\mathrm{~m}, 4 \mathrm{H}), 4.80(\mathrm{~d}, J=9.3 \mathrm{~Hz}, 1 \mathrm{H}), 4.97(\mathrm{~s}$, $1 \mathrm{H}), 7.10-7.30(\mathrm{~m}, 4 \mathrm{H}) ;{ }^{13} \mathrm{C}$ NMR $\left(75 \mathrm{MHz}, \mathrm{CDCl}_{3}\right): \delta$ (ppm) 20.4, 
31.9, 43.5, 48.4, 53.9, 57.3, 58.3, 70.9, 73.6, 97.9, 126.5, 127.9, 129.5, 131.9, 135.5, 140.9, 160.5; MS (ESI) $m / z 272\left([\mathrm{M}+\mathrm{H}]^{+}\right), 294$ $\left([\mathrm{M}+\mathrm{Na}]^{+}\right)$; HRMS (ESI) calcd for $\mathrm{C}_{17} \mathrm{H}_{21} \mathrm{NO}_{2}\left([\mathrm{M}+\mathrm{H}]^{+}\right)$: 272.1651; found: 272.1600 .

Compound 14b: $[\alpha]_{\mathrm{D}}^{23}=-90.4$ (c $\left.0.24, \mathrm{CHCl}_{3}\right) ;{ }^{1} \mathrm{H} \quad \mathrm{NMR}$ (300 MHz, $\left.\mathrm{CDCl}_{3}\right): \delta(\mathrm{ppm}) 1.60-1.85(\mathrm{~m}, 3 \mathrm{H}), 1.99-2.15(\mathrm{~m}, 2 \mathrm{H})$, 2.58-2.85 (m, 3H), 2.99-3.12 (m, 2H), 3.62-3.78 (m, $1 \mathrm{H}), 3.80(\mathrm{~s}$, $3 \mathrm{H}), 4.90(\mathrm{~d}, J=9.3 \mathrm{~Hz}, 1 \mathrm{H}), 4.98(\mathrm{~d}, J=9.3 \mathrm{~Hz}, 1 \mathrm{H}), 5.07(\mathrm{~s}, 1 \mathrm{H})$, 7.38 (d, $J=8.3 \mathrm{~Hz}, 1 \mathrm{H}), 7.40-7.60(\mathrm{~m}, 2 \mathrm{H}), 7.78(\mathrm{~d}, J=8.2 \mathrm{~Hz}, 1 \mathrm{H})$, $7.86(\mathrm{~d}, 1 \mathrm{H}, J=8.0 \mathrm{~Hz}), 8.18(\mathrm{~d}, J=8.1 \mathrm{~Hz}, 1 \mathrm{H}) ;{ }^{13} \mathrm{C} \mathrm{NMR}(75 \mathrm{MHz}$, $\mathrm{CDCl}_{3}$ ): $\delta$ (ppm) 20.1, 32.6, 44.1, 48.1, 49.9, 53.7, 57.3, 71.2, 73.1, 98.3, 122.6, 124.5, 126.4, 128.2, 128.8, 129.1, 129.9, 133.2, 133.8, 139.2, 160.7; HRMS (ESI) calcd for $\mathrm{C}_{21} \mathrm{H}_{23} \mathrm{NO}_{2}\left([\mathrm{M}+\mathrm{H}]^{+}\right)$: 322.1807; found: 322.1812 .

\subsection{Preparation of $(2 R, 5 S)$-2-hydroxy-3,4-dehydro-3- methoxy-15,16-desmethylenedioxycephalotaxane 15}

To a stirred $1 \mathrm{M}$ solution of $\mathrm{AlH}_{3}$ in anhydrous THF (31 equiv) (previously prepared from $\mathrm{LiAlH}_{4}$ and $\mathrm{AlCl}_{3}$ ) was added dropwise at $0{ }^{\circ} \mathrm{C}$ a $0.070 \mathrm{M}$ solution of compound 13 (1 equiv) in anhydrous THF and the mixture was stirred at $0{ }^{\circ} \mathrm{C}$ for $2.5 \mathrm{~h}$. The solution was then warmed to rt, quenched with a 2 M solution of Rochelle's salt and stirred for $15 \mathrm{~min}$. The aqueous phase was extracted twice with dichloromethane. The organic layers were combined, dried on magnesium sulfate, evaporated under reduce pressure and the residue obtained was purified by flash chromatography on silica gel with AcOEt/MeOH/NH $\mathrm{N}_{4} \mathrm{OH}(95 / 5 / 0.5 \mathrm{v} / \mathrm{v})$ as eluent to give compound $15(91 \%)$ as a colorless oil. Compound 15: ${ }^{1} \mathrm{H}$ NMR $\left(300 \mathrm{MHz}, \mathrm{CDCl}_{3}\right): \delta(\mathrm{ppm}) 1.48-1.68(\mathrm{~m}, 2 \mathrm{H}), 1.70-1.82(\mathrm{~m}, 3 \mathrm{H})$, 1.85-1.98 (m,1H), 2.25 (dd, $J=7.26,13.07 \mathrm{~Hz}, 1 \mathrm{H}), 2.70-2.82(\mathrm{~m}$, $1 \mathrm{H}), 2.85-2.94(\mathrm{~m}, 1 \mathrm{H}), 2.95-3.03(\mathrm{~m}, 1 \mathrm{H}), 3.05-3.12(\mathrm{~m}, 2 \mathrm{H})$, $3.32-3.48(\mathrm{~m}, 1 \mathrm{H}), 3.70(\mathrm{~s}, 3 \mathrm{H}), 4.72(\mathrm{dd}, J=4.62,7.22 \mathrm{~Hz}, 1 \mathrm{H})$, 7.10-7.25 (m, 4H); ${ }^{13} \mathrm{C}$ NMR (75 MHz, $\left.\mathrm{CDCl}_{3}\right): \delta(\mathrm{ppm}) 23.1,33.0$, 35.6, 46.1, 46.2, 50.5, 58.1, 71.2, 72.8, 119.0, 125.4, 127.1, 129.2, 130.8, 133.5, 138.7, 155.1; MS (ESI) $\mathrm{m} / \mathrm{z} 272\left([\mathrm{M}+\mathrm{H}]^{+}\right), 294$ $\left([\mathrm{M}+\mathrm{Na}]^{+}\right)$; HRMS (ESI) calcd for $\mathrm{C}_{17} \mathrm{H}_{21} \mathrm{NO}_{2}\left([\mathrm{M}+\mathrm{H}]^{+}\right)$: 272.1651; found: 272.1660 .

\section{Acknowledgments}

The CNRS and the French Ministry of Education and Research are acknowledged for funding.

\section{References}

1. Paudler, W. W.; Kerley, G. I.; McKay, J. J. Org. Chem. 1963, 28, 2194-2197.

2. (a) Powell, R. G.; Weisleder, D.; Smith, C. R., Jr; Rohwedder, W. K. Tetrahedron Lett. 1970, 11, 815-818; (b) Jilal Miah, M. A.; Hudlicky, T.;
Reed, J. W.. In The Alkaloids; Cordell, G. A., Ed.; Academic Press: San Diego, 1998; Vol. 51, pp 199-264.

3. For recent papers on clinical trials of homoharringtonine, see: (a) Kantarjian, $H$ M.; Talpaz, M.; Santini, V.; Murgo, A.; Cheson, B.; O'Brien, S. M. Cancer 2001, 92, 1591-1605; (b) O’Brien, S. M.; Talpaz, M.; Cortes, J.; Shan, J.; Giles, F. J.; Faderl, S.; Thomas, D.; Garcia-Manero, G.; Mallard, S.; Rios, M. B.; Koller, C.; Kornblau, S.; Andreeff, M.; Murgo, A.; Keating, M.; Kantarjian, H. M. Cancer 2002, 94 2024-2032; (c) Scappini, B.; Onida, F.; Kantarjian, H. M.; Dong, L.; Verstovsek, S.; Keating, M. J.; Beran, M. Cancer 2002, 94, 2653-2662; (d) Hitt, E. Lancet Oncol. 2002, 3, 259; (e) Quintas-Cardama, A.; Cortes, J. Expert Opin. Pharmacother. 2008, 9, 1029-1037; (f) Zhang, W. G.; Wang, F. X.; Chen, Y. X. Cao, X. M.; He, A. L.; Liu, J.; Ma, X. R.; Zhao, W. H.; Liu, S. H.; Wang, J. L. Am. J. Hematol. 2008, 83, 185-188; (g) Quintás-Cardama, A.; Kantarjian, H.; Cortes, J. Cancer 2009, 115, 5382-5393.

4. Racemic total syntheses: (a) Auerbach, J.; Weinreb, S. M. J. Am. Chem. Soc. 1972 94, 7172-7173; (b) Semmelhack, M. F.; Chong, B. P.; Jones, L. D. J. Am Chem Soc. 1972, 94, 8629-8630; (c) Semmelhack, M. F.; Chong, B. P.; Stauffer, R. D. Rogerson, T. D.; Chong, A.; Jones, L. D. J. Am. Chem. Soc. 1975, 97, 2507-2516; (d) Weinreb, S. M.; Auerbach, J. J. Am. Chem. Soc. 1975, 97, 2503-2506; (e) Burkholder, T. P.; Fuchs, P. L. J. Am. Chem. Soc. 1988, 110, 2341-2342; (f) Kuehne, M. E.; Bornmann, W. G.; Parsons, W. H.; Spitzer, T. D.; Blount, J. F.; Zubieta, J. J. Org. Chem. 1988, 53, 3439-3450; (g) Burkholder, T. P.; Fuchs, P. L. J Am.Chem. Soc. 1990, 112, 9601-9613; (h) Ishibashi, H.; Okano, M.; Tamaki, H. Maruyama, K.; Yakura, T.; Ikeda, M. J. Chem. Soc., Chem. Commun. 1990, 14361437; (i) Ikeda, M.; Okano, M.; Kosaka, K.; Kido, M.; Ishibashi, H. Chem. Pharm. Bull. 1993, 41, 276-281; (j) Lin, X.; Kavash, R. W.; Mariano, P. S. J. Am. Chem. Soc. 1994, 116, 9791-9792; (k) Lin, X.; Kavash, R. W.; Mariano, P. S. J. Org. Chem. 1996, 61, 7335-7347; (1) Tietze, L. F.; Schirok, H. Angew. Chem., Int. Ed. 1997, 36, 1124-1125; (m) Koseki, Y.; Sato, H.; Watanabe, Y.; Nagasaka, T. Org. Lett. 2002 4, 885-888; (n) Suga, S.; Watanabe, M.; Yoshida, J. J. Am. Chem.Soc. 2002, 124 14824-14825; (o) Li, W.-D. Z.; Wang, Y.-Q. Org. Lett. 2003, 5, 2931-2934; (p) Li, W.-D. Z.; Ma, B.-C. J. Org. Chem. 2005, 70, 3277-3280; (q) Ma, B.-C.; Wang, Y.-Q.; Li, W.-D. Z. J. Org. Chem. 2005, 70, 4528-4530; (r) Li, W.-D. Z.; Wang, X.-W. Org. Lett. 2007, 9, 1211-1214; Enantioselective total syntheses: (s) Zhong, S.; Liu, W.; Ling, Y.; Li, R. Zhongguo Yaowu Huaxue Zazhi 1994, 4, 84; (t) Isono, N.; Mori, M. J. Org.Chem. 1995, 60, 115-119; (u) Nagasaka, T.; Sato, H.; Saeki, S.-I. Tetrahedron: Asymmetry 1997, 8, 191-194; (v) Ikeda, M.; El Bialy, S. A. A.; Hirose, K.-I.; Kotake, M.; Sato, T.; Bayomi, S. M. M.; Shehata, I. A.; Abdelal, A. M.; Gad, L. M.; Yakura, T. Chem. Pharm. Bull. 1999, 47, 983-987; (w) Tietze, L. F.; Schirok, H. J. Am. Chem. Soc. 1999, 121, 10264-10269; (x) El Bialy, S. A. A.; Ismail, M. A.; Gad, L. M.; Abdelal, A. M. M. Med. Chem. Res. 2002, 11, 293; (y) Eckelbarger, J. D.; Wilmot, J. T.; Gin, D. Y. J. Am. Chem. Soc. 2006, 128, 1037010371; (aa) Liu, Q.; Ferreira, E. M.; Stoltz, B. M. J. Org. Chem. 2007, 72, 73527358; (bb) Esmieu, W. R.; Worden, S. W.; Catterick, D.; Wilson, C.; Hayes, C. J. Org. Lett. 2008, 10, 3045-3048; (cc) Taniguchi, T.; Ishibashi, H. Org. Lett. 2008, 10, 4129-4131.

5. Planas, L.; Pérard-Viret, J.; Royer, J. J. Org. Chem. 2004, 69, 3087-3092.

6. Mikolajczak, K. L.; Smith, C. R.; Weisleder, D. J. Med. Chem. 1977, 20, 328-332. See also Ref. $2 b$.

7. Tietze, L. F.; Braun, H.; Steck, P. L.; El Bialy, S. A. A.; Tölle, N.; Düfert, A. Tetrahedron 2007, 63, 6437-6445.

8. Planas, L.; Pérard-Viret, J.; Royer, J.; Selkti, M.; Thomas, A. Synlett 2002, 10 , 1629-1632.

9. (a) Baussanne, I.; Royer, J. Tetrahedron Lett. 1996, 37, 1213-1216; (b) Baussanne, I.; Schwardt, O.; Royer, J.; Pichon, M.; Figadère, B.; Cave, A Tetrahedron Lett. 1997, 38, 2259-2262; (c) Baussanne, I.; Travers, C.; Royer, J. Tetrahedron: Asymmetry 1998, 9, 797-804.

10. Ito, Y.; Hirao, T.; Saegusa, T. J. Org. Chem. 1978, 43, 1011-1013.

11. Wilds, A. L. Org. Reaction 1944, 2, 178. 